\title{
An Assessment of the Reporting Pattern of Adverse Events Following Immunizations in VigiAccess
}

\author{
Peter Yamoah ${ }^{1,2,3}$ \& Frasia Oosthuizen ${ }^{2}$ \\ ${ }^{1}$ Komfo Anokye Teaching Hospital, Ghana \\ ${ }^{2}$ College of Health Sciences, University of KwaZulu Natal, Durban, South Africa \\ ${ }^{3}$ Department of Pharmacy Practice, Faculty of Pharmacy and Pharmaceutical Sciences, Kwame Nkrumah \\ University of Science and Technology, Ghana \\ Correspondence: Peter Yamoah, Box KS13055, Kumasi, Ghana. Tel: 233-24-337-4250. E-mail: \\ pyamoah@gmail.com
}

Received: July 29, 2018 Accepted: August 17, 2018 Online Published: October 13, 2018

doi:10.5539/gjhs.v10n11p46 URL: https://doi.org/10.5539/gjhs.v10n11p46

\begin{abstract}
Purpose: Globally, adverse events following immunization (AEFI) reporting continues to be a challenge. It is estimated that about $95 \%$ of AEFIs never get reported after vaccinations necessitating strategies to improve it. The introduction of databases such as VigiAccess in which AEFI data from Pharmacovigilance centres around the world can be assessed is an important step towards improving AEFI reporting and enhancing vaccine safety. This study assessed the reporting pattern of AEFIs from the various continents of the world in VigiAccess, an open-access pharmacovigilance database.
\end{abstract}

Methods: VigiAccess was thoroughly searched on the $5^{\text {th }}$ of February 2018 for the categories of reported AEFIs and number and types of AEFIs reported for measles vaccine, oral polio vaccine, yellow fever vaccine, pneumococcal vaccine, rotavirus vaccine, meningococcal vaccine, tetanus vaccine and BCG vaccine.

Results: After a thorough search through VigiAccess, 27 categories of reported AEFIs were retrieved. The total number of AEFIs for the 8 vaccines was 813,973. General disorders and administration site conditions were the highest number of AEFIs (251,405 representing 30.9\%) followed by skin and subcutaneous tissue disorders (93,011 representing $11.4 \%$ ) and nervous system disorders (89,077 representing 10.9\%). With the continental data, the Americas recorded the highest number of AEFIs followed by Europe, Oceania, Asia and Africa.

Conclusion: General and vaccine administration site conditions were the highest number of AEFIs. The Americas recorded the highest number of AEFIs whereas Africa recorded the least. VigiAccess needs improvement in data synchronization to enhance its reliability.

Keywords: pharmacovigilance, adverse event following immunization, Uppsala Monitoring Centre, VigiAccess

\section{Introduction}

\subsection{Background}

The importance of vaccines in preventing deaths resulting from infectious diseases cannot be overemphasized (Lei, Balakrishnan, Gidudu, \& Zuber, 2018). Globally, it is estimated that about 2.5 million child deaths alone are prevented by vaccines annually (World Health Organization, 2009). Out of these preventable deaths, a larger proportion is likely to occur in low and middle income countries (LMIC) where more doses of vaccines are used compared to the developed world (WHO, 2012a). As more doses of vaccines are administered, the risk of adverse events following immunization also increases. There is therefore the need to intensify the reporting of AEFIs particularly in LMIC in order to improve vaccine safety.

Vaccines are potent agents which are rigorously tested before approval for disease prevention (Miller, Moro, Cano \& Shimabukuro, 2015). However some adverse events associated with them manifest after being used in larger populations following their approval some of which could be fatal if not managed promptly (Chung, 2014; Erlewyn-lajeunesse, Bonhoeffer, Ruggeberg, \& Heath, n.d.). Globally, there have been concerns about vaccine safety which have led to dwindling confidence in immunization programs due to news about aftermath of rare but serious AEFIs such as hospitalization and death (Ozawa \& Stack, 2013). A classical example is the flaw in the 
manufacture of the Salk polio vaccine (improper inactivation of the virus) which led to 40,000 active polio cases causing 51 cases of permanent paralysis and five deaths among vaccinees, and 113 cases of paralysis and five deaths among contacts of vaccinated individuals (Offit, 2005).

To enhance confidence of potential vaccinees in immunization programs it is of utmost importance to develop strategies to document and report AEFIs. A study conducted in 2006 found out that about $95 \%$ of AEFIs never get reported after vaccination (Hazell \& Shakir, 2006). Current studies have also observed similar trends of reporting. This usually happens because healthcare professionals to whom these AEFIs are reported usually feel that most of the AEFIs are mild and not harmful (Danova, Kocourkova, \& Celko, 2017). Moreover, HCPs are more likely to report AEFIs they are already familiar with than unexpected events (Parrella, Braunack-Mayer, Gold, Marshall, \& Baghurst, 2013). In a study conducted in the Czech Republic comparing observed AEFIs from a sample of paediatric GP practices with officially reported rate for instance, it was found out that the officially reported rate was far lower than that observed in the study (Danova et al., 2017). This necessitates the education of healthcare professionals on the need to report any unusual events after immunization regardless of how mild they may appear.

AEFI reporting could be active or passive. In active AEFI reporting, electronic systems are used to monitor AEFIs whereas passive reporting encompasses the voluntary reporting of AEFI by healthcare professionals, patients and the general public (Cashman et al., 2017; Li et al., 2014; Parrella, Gold, Braunack-Mayer, Baghurst, \& Marshall, 2014). The vast majority of AEFI reports to national pharmacovigilance centres is passive in nature and may have some limitations such as the difficulty to link an adverse event to a vaccine, under-reporting of less severe AEFIs, misdiagnosis of AEFIs and inability to capture late occurring AEFIs after vaccination (Hu et al., 2013; Parrella et al., 2014). Despite these shortcomings, passive AEFI data especially from the public is important to collect in order for them to feel included in vaccine safety monitoring. Moreover, since the public is more likely to report unexpected AEFIs than HCPs their reports could lead to the discovery of rare AEFIs (Clothier et al., 2014; Parrella et al., 2013). Due to this, several studies have suggested that vaccinees and their caretakers (in case of children) must be well equipped to actively partake in AEFI reporting (Hazell, Cornelius, Hannaford, Shakir, \& Avery, 2013; Inch, Watson, \& Anakwe-Umeh, 2012).

Pharmacovigilance centres are mandated by the WHO to submit adverse drug reaction reports including AEFI reports from various countries to a large pool of adverse drug reaction electronic database called Vigibase (Ampadu et al., 2016). The database system includes the International Conference on Harmonization (ICH) E2B compatible Independent Case Safety Reports (ICSRs) database, the WHO Drug Dictionaries (WHO-DD and -DDE), WHO Adverse Reaction Terminology (WHO-ART), International Classification of Diseases (ICD), and the Medical Dictionary for Regulatory Activities (MedDRA) (Lindquist, 2008). Vigibase categorizes safety data into sex, disease condition for which a particular medicine was given as well as other relevant features on ICSR forms on which safety data of patients are recorded before submission. These features make the database a good resource for the UMC and other stakeholders of pharmacovigilance as it gives an indication of the number of safety reports submitted by each country from the time they joined the PIDM. Vigibase also gives information about the safety profile of medicines and vaccines as well as the quality of reports from the various reporting institutions to the national pharmacovigilance centres (Ampadu et al., 2016). Vigibase may also have the additional advantage of other adverse drug reactions and AEFIs which may not be known in published data as some ADRs and AEFIs are very rare, unsuspecting and late occurring (Yadav, 2008). Despite the relevant information in Vigibase, it is unavailable to the general public. However, the WHO has an alternative database called VigiAccess which is open to the general public and serves as a repository of reported adverse drug reactions and AEFIs (Shankar, 2016). The disadvantage of VigiAccess data is the fact that it does not include country specific adverse reaction and AEFI data but rather, continental adverse drug reaction and AEFI data. Additionally, details of patient data on ICSR forms are also not available in VigiAccess. Despite these disadvantages, VigiAccess can serve as a powerful tool for quick reference of adverse drug reactions and AEFIs by both HCPs and the general public due to its open access nature. This study assessed the reporting pattern of AEFIs from the various continents of the world in VigiAccess. It is hoped that findings from the study will serve as baseline data based on which future researchers will build upon towards improving vaccine safety and the quality of VigiAccess data.

\subsection{Study Aim}

To assess the reporting pattern of AEFIs in VigiAccess

\subsection{Study Objectives}

1). To ascertain the categories of reported AEFIs in VigiAccess

2). To quantify the number of the various categories of AEFI in VigiAccess for some selected vaccines 
3). To quantify the total number of AEFIs of some selected vaccines in VigiAccess on continental basis

4). To assess the limitations to the use of VigiAccess

\section{Methods}

\subsection{Study Design}

The study employed a secondary research design in that existing AEFI data from a database was analyzed and interpreted.

\subsection{Data Source}

The WHO open access database for reported adverse drug reactions, VigiAccess was the data source for this study. VigiAccess contains adverse drug reaction and AEFI data of 131 full member countries of the WHO Program for International Drug Monitoring [PIDM] (UMC, 2018). The database contains the cumulative number of AEFIs of various vaccines from various countries from the time they joined the PIDM. VigiAccess was thoroughly searched on the $5^{\text {th }}$ of February 2018 for the categories of reported AEFIs. Another search was conducted on the types and number of AEFIs reported for measles vaccine, oral polio vaccine, yellow fever vaccine, pneumococcal vaccine, rotavirus vaccine, meningococcal vaccine, tetanus vaccine and BCG vaccine. These vaccines were randomly selected from a list of 26 vaccines from the website of the Centres for Disease Control and Prevention that are commonly used across the various continents of the world for disease control and prevention (CDC, 2018).

\subsection{Data Analysis}

Data was periodically entered into SPSS software version 21 and analyzed after full entry. The categories of AEFI were classified based on the body systems and the vaccine product as done at the VigiAccess data interface of the VigiAccess website. The number of AEFIs reported for the vaccines were categorized based on the continents of the world (i.e. Africa, Asia, the Americas, Europe and Oceania). Furthermore AEFI data was categorized based on populations of the various continents of the world to compare population and number of AEFI reports. Tables were used to summarize the categories of AEFI and continental AEFI data and a graph was used to elucidate continental data.

\section{Results}

\subsection{Categories of AEFIS}

After a thorough search through VigiAccess, 27 categories of reported AEFIs in the database were retrieved. These included blood and lymphatic system disorders, cardiac disorders, congenital, familial and genetic disorders, ear and labyrinth disorders, endocrine disorders, eye disorders, gastrointestinal disorders, vaccine administration site conditions, hepatobiliary disorders, immune system disorders, infections and infestations, injury, poisoning and procedural complications, metabolism and nutrition disorders, musculoskeletal and connective tissue disorders, neoplasms (benign, malignant and unspecified such as cysts and polyps), nervous system disorders, pregnancy, puerperium and perinatal conditions, psychiatric disorders, renal and urinary disorders, reproductive system and breast disorders, respiratory, thoracic and mediastinal disorders, skin and subcutaneous tissue disorders, social circumstances, surgical and medical procedures and vascular disorders.

\subsection{Vaccines versus AEFI Categories}

The numbers of vaccinees affected by the various AEFI categories for each vaccine are illustrated in Table 1

From Table 1, the total number of AEFIs for the 8 vaccines was 813,973 . General disorders and administration site conditions were the highest number of AEFIs (251,405 representing 30.9\%) followed by skin and subcutaneous tissue disorders (93,011 representing 11.4\%) and nervous system disorders (89,077 representing 10.9\%).

PCV accounted for the highest number of AEFIs (317,208 representing 39.0\%) followed by OPV $(185,829$ representing 22.8\%) and MCV (145,447 representing 17.9\%). Pneumococcal vaccine and OPV accounted for more than half of the total number of cardiac, congenital, endocrine, eye, general disorders, immune system disorders, infections and infestations, hepatotoxicity, metabolic and nutrition disorders, social circumstances and surgical and medical procedures related AEFIs. The pneumococcal and meningococcal vaccines also accounted for over a half of ear and labyrinth disorders. Additionally over a half of AEFIs associated with the nervous system, pregnancy, and vascular disorders were associated with the pneumococcal vaccine, OPV and meningococcal vaccine whereas over half of the gastrointestinal AEFIs were caused by pneumococcal vaccine, rotavirus vaccine, meningococcal vaccine and OPV. Furthermore, pneumococcal and rotavirus vaccines accounted for over half of injury, poisoning and procedural complications related AEFIs. The BCG vaccine alone caused over a half of lymphatic system related AEFIs. Measles vaccine was the only vaccine that recorded no vaccine product related 
AEFIs.

Table 1. Number of reported AEFI categories for the various vaccines

\begin{tabular}{|c|c|c|c|c|c|c|c|c|c|}
\hline \multirow{2}{*}{ AEFI } & \multicolumn{9}{|c|}{ Vaccine $(\mathbf{N})$} \\
\hline & MV & OPV & YFV & PCV & $\mathbf{R V}$ & MCV & TV & BCG & TOTAL \\
\hline Blood and lymphatic system disorders & 204 & 1799 & 391 & 3456 & 605 & 1282 & 330 & 8561 & 16628 \\
\hline Cardiac disorders & 95 & 2861 & 232 & 3950 & 971 & 1221 & 192 & 162 & 9684 \\
\hline $\begin{array}{l}\text { Congenital, familial and genetic } \\
\text { disorders }\end{array}$ & 11 & 238 & 29 & 345 & 186 & 55 & 18 & 28 & 910 \\
\hline Ear and labyrinth disorders & 36 & 415 & 172 & 806 & 77 & 680 & 126 & 28 & 2340 \\
\hline Endocrine disorders & 1 & 53 & 23 & 95 & 15 & 45 & 18 & 13 & 263 \\
\hline Eye disorders & 161 & 2728 & 448 & 3503 & 813 & 2657 & 227 & 200 & 10737 \\
\hline Gastrointestinal disorders & 537 & 10779 & 2615 & 17448 & 14722 & 12384 & 1130 & 624 & 60239 \\
\hline $\begin{array}{l}\text { General disorders and administration } \\
\text { site conditions }\end{array}$ & 2493 & 63617 & 6966 & 105680 & 13039 & 43342 & 9268 & 7000 & 251405 \\
\hline Hepatobiliary disorders & 21 & 174 & 165 & 404 & 122 & 95 & 35 & 224 & 1240 \\
\hline Immune system disorders & 519 & 2286 & 340 & 3454 & 407 & 1453 & 612 & 223 & 9294 \\
\hline Infections and infestations & 797 & 12702 & 798 & 20631 & 5332 & 4225 & 778 & 10233 & 55496 \\
\hline $\begin{array}{l}\text { Injury, poisoning and procedural } \\
\text { complications }\end{array}$ & 198 & 2416 & 345 & 8901 & 5554 & 4816 & 332 & 726 & 23288 \\
\hline Investigations & 121 & 4953 & 829 & 18047 & 7257 & 5641 & 476 & 560 & 37884 \\
\hline Metabolism and nutrition disorders & 104 & 3424 & 254 & 5826 & 2833 & 1758 & 128 & 161 & 14488 \\
\hline $\begin{array}{l}\text { Musculoskeletal and connective } \\
\text { tissue disorders }\end{array}$ & 196 & 4243 & 1950 & 17787 & 730 & 8483 & 1656 & 962 & 36007 \\
\hline $\begin{array}{l}\text { Neoplasms benign, malignant and } \\
\text { unspecified }\end{array}$ & 7 & 123 & 27 & 251 & 39 & 71 & 30 & 225 & 773 \\
\hline Nervous system disorders & 1139 & 21379 & 4326 & 29007 & 5153 & 24450 & 2536 & 1087 & 89077 \\
\hline $\begin{array}{l}\text { Pregnancy, puerperium and perinatal } \\
\text { conditions }\end{array}$ & 19 & 98 & 78 & 91 & 28 & 98 & 33 & 10 & 455 \\
\hline Product issues & 0 & 21 & 5 & 108 & 51 & 134 & 8 & 71 & 398 \\
\hline Psychiatric disorders & 196 & 15067 & 364 & 14412 & 4855 & 4362 & 311 & 212 & 39779 \\
\hline Renal disorders & 25 & 429 & 155 & 814 & 151 & 477 & 84 & 920 & 3055 \\
\hline $\begin{array}{l}\text { Reproductive system and breast } \\
\text { disorders }\end{array}$ & 3 & 94 & 75 & 235 & 24 & 168 & 30 & 186 & 815 \\
\hline $\begin{array}{l}\text { Respiratory, thoracic and mediastinal } \\
\text { disorders }\end{array}$ & 318 & 6580 & 918 & 11630 & 2474 & 3905 & 468 & 535 & 26828 \\
\hline $\begin{array}{l}\text { Skin and subcutaneous tissue } \\
\text { disorders }\end{array}$ & 1720 & 21782 & 2535 & 39401 & 4368 & 18150 & 3174 & 1881 & 93011 \\
\hline Social circumstances & 2 & 118 & 63 & 713 & 85 & 340 & 47 & 43 & 1411 \\
\hline Surgical and medical procedures & 8 & 675 & 85 & 2743 & 1943 & 472 & 55 & 232 & 6213 \\
\hline Vascular disorders & 131 & 6775 & 389 & 7470 & 1679 & 4683 & 679 & 449 & 22255 \\
\hline Total & 9062 & 185829 & 24577 & 317208 & 73513 & 145447 & 22781 & 35556 & 813973 \\
\hline
\end{tabular}

Key: MV- Measles vaccine, OPV-Oral Polio vaccine, YFV-Yellow Fever vaccine, PCV-Pneumococcal vaccine, RVRotavirus vaccine, MCV- Meningococcal vaccine, TV-Tetanus vaccine and BCG- Bacillus Calmette Guerin vaccine. 


\subsection{Continental AEFI Reports}

In the VigiAccess software, the global population is classified based on the five continents of the world including Africa, Americas (north and south), Asia, Europe and Oceania. With the continental data, the Americas recorded the highest number of AEFIs since joining the WHO PIDM program, followed by Europe, Oceania, Asia and Africa. Africa recorded the highest number of yellow fever vaccine AEFIs, the Americas recorded the highest number of OPV, pneumococcal and rotavirus AEFIs, and Europe recorded the highest number of both meningococcal and Tuberculosis (BCG) vaccine AEFIs. Table 2 summarizes the distribution of AEFIs across the various continents of the world.

Table 2. AEFI reports from the various continents of the world

\begin{tabular}{lllllll}
\hline \multirow{2}{*}{ Vaccine } & \multicolumn{7}{c}{ Continent } & Asia & Europe & Oceania & Total \\
\cline { 2 - 6 } & Africa & Americas & Asia & N & N \\
\hline Measles & 573 & 2310 & 364 & 1685 & 212 & 5144 \\
Oral Polio & 1915 & 79068 & 4645 & 4902 & 1707 & 92237 \\
Yellow fever & 5290 & 4244 & 124 & 2499 & 243 & 12400 \\
Pneumococcal & 825 & 97103 & 5848 & 34082 & 9591 & 147449 \\
Rotavirus & 312 & 18242 & 3073 & 6070 & 3850 & 31547 \\
Meningococcal & 2862 & 29063 & 309 & 34364 & 5412 & 72010 \\
Tetanus & 197 & 4262 & 897 & 7091 & 545 & 12992 \\
Tuberculosis (BCG) & 351 & 3213 & 4235 & 16958 & 621 & 25378 \\
Total (\%) & $12325(3.1)$ & $237505(59.5)$ & $19495(4.9)$ & $107651(27.0)$ & $22181(5.6)$ & 399157 \\
\hline
\end{tabular}

\subsection{Comparison of AEFIs With Current Continental Population}

On the $5^{\text {th }}$ of February 2018, when VigiAccess was searched, Asia had the highest population in the world, followed by Africa, the Americas, Europe and Oceania (World Population Review, 2018). Comparing the current population of the various continents with the number of AEFIs associated with the vaccines in this study, 4 in a million AEFI reports were from Asia, 10 in a million AEFI reports were from Africa, 1 in 10,000 AEFI reports were from Europe, 3 in 10,000 AEFI reports were from America and 5 in 10,000 AEFI reports were from Oceania. Table 3 illustrates the comparison of AEFIs across continents of the world.

Table 3. AEFIs versus current continental populations

\begin{tabular}{lllll}
\hline Continent & Population & $\begin{array}{l}\text { Percentage } \\
\text { population (\%) }\end{array}$ & Number of AEFIs & AEFIs per citizen \\
\hline Africa & $1,281,791,015$ & 17.4 & 12325 & $9.6^{*} 10^{-6}$ \\
Americas & $754,587,688$ & 10.2 & 237505 & $3.1 * 10^{-4}$ \\
Asia & $4,545,133,094$ & 61.7 & 19495 & $4.2^{*} 10^{-6}$ \\
Europe & $742,543,873$ & 10.1 & 107651 & $1.4^{*} 10^{-4}$ \\
Oceania & $41,157,193$ & 0.6 & 22181 & $5.3^{*} 10^{-4}$ \\
\hline
\end{tabular}

In comparing population with AEFI reports, Oceania therefore ranks first in reporting followed by the Americas, Europe, Africa and Asia respectively.

\section{Discussion}

\subsection{Categories of Reported AEFIs}

An AEFI is any untoward medical occurrence that may present after the administration of a vaccine but which does not necessarily have a causal relationship with the treatment which could be any unfavourable or unintended sign, 
abnormal laboratory finding, symptom or disease (CIOMS, 2012). The categories of AEFIs reported as revealed by the search in VigiAccess involved all body systems such as the cardiovascular, respiratory, nervous, skeletal, immune, circulatory, renal, reproductive, endocrine systems, eyes, ears and the skin. AEFIs typically result from immune reactions following immunization and can affect virtually all systems of the body. In all cases, causality assessment is necessary to be conducted on serious AEFIs to establish whether the medical occurrence resulted from the vaccine or not (Williams et al., 2013). However, this may not be necessary in minor AEFIs such as pain, swelling and redness of site of vaccination which are almost always expected after every vaccination. The minor reactions usually occur few hours after vaccination, resolve after a short period of time and poses little danger to the vaccinees (CIOMS, 2012). When properly done, causality assessment could help curb under-reporting and over-reporting of AEFIs. This means that healthcare providers and regulatory authorities must have cutting edge knowledge in the diagnosis and causality assessment of AEFIs. It is claimed that existing disease conditions before immunizations could trigger AEFIs. However, such claims could be verified through safety monitoring or clinical studies to be sure whether the immune response was actually caused by the vaccine (Shimabukuro et al., 2015). For instance, following the emergence of the H1N1 pandemic, mass public vaccination with AS03-adujvanted A (H1N1) pdm09 vaccine was undertaken in Sweden during which many narcolepsy cases were reported (Lakemedelsverket, 2011). Based on available information in the literature at the time, this led to a divided opinion among health experts as to whether the narcolepsy was immune mediated resulting from infections such as streptococcal and H1N1 infection (Aran et al., 2009) or vaccine induced (Han et al., 2011). Consequently a case-control study was conducted to ascertain the actual cause of the narcolepsy. The findings of the study did not support a disease history of narcolepsy before the $\mathrm{A}(\mathrm{H} 1 \mathrm{~N} 1) \mathrm{pdm} 09$ vaccination and therefore led to the diagnosis of the medical occurrence as a vaccine related AEFI (Lamb et al., 2016).

\subsection{Vaccines versus Number of AEFIs}

The top 3 AEFIs caused by the vaccines studied were general disorders and administration site conditions, skin and subcutaneous tissue disorders and nervous system disorders. General and nervous system disorders are mainly caused by the immunological response to vaccines. While general disorders are mostly self limiting, neurological disorders are among the most serious, and on rare occasions, life-threatening complications after vaccination (Miravalle, Biller, \& Bonwit, 2010). Even though life-threatening neurological cases after vaccination are rare, causality assessment to ensure that there was no pre-existing neurological condition before vaccination is of the essence if it occurs and specialist neurologist care may be appropriate to avert any unforeseen danger to vaccinees (Williams et al., 2011). Administration site conditions and subcutaneous tissue disorders are caused by reaction to components of the vaccines and wrong vaccination technique by the healthcare provider administering the vaccine. Patient profiling to access whether they react fatally to any of the components of the vaccine must be done prior to any vaccination exercise (Chung, 2014). Wrong vaccination techniques such as injecting at the wrong site have often led to abscesses and paralysis when nerves get damaged in the process. It is therefore important that vaccinators are educated periodically on vaccination techniques to prevent these occurrences (Lussier et al., 1999).

The top three vaccines associated with more than half of the recorded AEFIs were pneumococcal, oral polio and meningococcal vaccines. Each of these vaccines recorded general and administration site disorders as the highest number of AEFIs associated with its administration. It is therefore necessary for vaccinators to administer these 3 vaccines in particular with caution. Concurrent administration of these vaccines therefore calls for careful evaluation of risks and benefits as their additive effects could pose a risk to the vaccinees. Pneumococcal vaccine is indicated in patients with sickle cell disease, HIV infection and asthma for prophylaxis against pneumonia in many countries (Crum-Cianflone \& Wallace 2014; Han et al., 2015). A study conducted by Han et al., 2015 observed that pneumococcal vaccine (PPSV23) could cause many severe adverse reactions when administered to paediatric and adolescent sickle cell disease patients even though not as much as it does in HIV and asthma patients. The authors suggested that it may be prudent for healthcare providers not to simultaneously administer pneumococcal vaccine (PPSV23) with other vaccines in order not to potentiate AEFIs associated with the pneumococcal vaccine.

\subsection{AEFI Reporting Across Continents}

The results showed that about $60 \%$ of all AEFIs worldwide were reported by the Americas whereas the least number was reported by Africa. These findings were similar to a June 2015 search in Vigibase. In the 2015 search however, reports from Africa were less than $1 \%$ as against $3.1 \%$ in this study. AEFI reports from the other continents in the 2015 search were America-60\%, Europe- $28 \%$, Oceania- $6 \%$ and Asia-5\% (UMC, 2015). Even though these results show an improvement in number of AEFIs from Africa, there is still room for improvement. Comparing continental populations per AEFI report, Oceania ranked highest whilst Asia ranked lowest. While it may not be fair to compare AEFIs from continents because various vaccines have been used to different extents 
across different continents from the time AEFI reporting began, it is also worth mentioning that since more doses of vaccines are currently being administered in low and middle income countries than developed countries, more AEFI reports are expected from low and middle income countries (WHO, 2012b, 2012c). Africa and Asia together reported about $8 \%$ of the global AEFIs. As Africa and Asia contribute to over three quarters of the population of the world, the largest proportion of the world's total AEFI reports was expected to be reported from there. Given the fact that more vaccine doses are administered in most countries in these 2 continents due to the large proportion of low and middle income countries, more needs to be done in maximizing AEFI reporting (WHO, 2012a). These continents need to expand the scope of AEFI reporting to all its countries as well as build capacity of reporting in order for number of AEFIs to be commensurate with the large number of vaccine doses administered.

\subsection{Overview of AEFI Reporting and Strengths of Study}

This study is the first of its kind to demonstrate the utility of AEFI data in VigiAccess as well as to identify the challenges associated with such data which could be improved in the bid to further vaccine safety.

Even though there has been an improvement in the safety monitoring of vaccines from the beginning of the $21^{\text {st }}$ century, through functional safety monitoring systems there is still room for improvement (Chen et al., 2015). Since the establishment of the WHO Program for International Drug Monitoring (PIDM) in 1968, AEFI reporting had been very slow until the introduction of rigorous monitoring and reporting mechanisms by the WHO and national pharmacovigilance centres from the beginning of the $21^{\text {st }}$ century. These included the Global Vaccine Action Plan (GVAP) and the WHO/UNICEF Joint Reporting Form (JRF) on immunization among others (WHO, 2015; WHO, 2016). GVAP and the WHO/UNICEF JRF for instance recommend the rigorous monitoring of AEFIs by countries and have identified the AEFI reporting ratio (number of AEFI reports per 100,000 surviving infants) as a key indicator for measuring the success of immunization programs. This has challenged many countries to improve vaccine safety in infants.

As of 2010, low and middle income countries were still lagging behind in vaccine safety and AEFI reporting, leading to the development of the Global Vaccine Safety Blueprint by the WHO and its strategic partners in 2011 (WHO, 2012b, 2012c). The goal of the blueprint was to further improve the capacity of regulatory authorities and other stakeholders of pharmacovigilance in low and middle income countries. To augment the efforts of the WHO and its partners in the improvement of vaccine safety, it is important for stakeholders to be abreast with the current situation through regular updates from the WHO and regulatory authorities. Stakeholders of vaccine safety include but not limited to healthcare professionals, academic institutions, vaccine manufacturers, the media, politicians, policy makers, expanded program on immunization (EPI) managers, non-governmental organizations and the general public (Hardt et al., 2013; National Academy of Sciences, 2013). All these groups play unique and significant roles towards improving vaccine safety by way of advocacy, research, reporting and policy formulation on AEFIs. VigiAccess is a good source of information on AEFIs of vaccines and adverse drug reactions (ADRs) in general which stakeholders can easily fall upon for current updates because of its open access nature. Academicians can analyze data in VigiAccess to inform politicians and policy makers on trends in AEFI reporting and ways to improve it. Moreover, vaccine manufacturers could be informed through VigiAccess as to vaccine products and their associated serious AEFIs which could lead to product safety improvement or recalls. Additionally all other stakeholders could join advocates in spreading information on vaccines and their associated AEFIs and the need for reporting.

\subsection{Limitations of VigiAccess}

In this study, the analysis of the various categories of AEFI in VigiAccess yielded a total number of 813,973 whereas the AEFIs from the various continents were 399,157. This disparity is a major limitation of the study. The disparity raises the concern as to which of the two figures to rely upon for informed decisions or policy formulation on vaccine safety. Even though the disparity did not have an effect on the continental analysis as it affected all continents to the same extent, it would have been better for both data to be same for better comparison between the two. This disparity has been explained at the VigiAccess website as due to the detection and removal of suspected duplicate reports from the VigiAccess dataset by an automatic algorithm called VigiMatch (VigiAccess Q \& A, $\mathrm{n}: \mathrm{d})$. VigiMatch includes only reports which are complete and deletes those with omitted information on ICSRs from the various continents. Moreover, for suspected duplicate reports only the most complete reports are used in the statistics in VigiAccess. Furthermore, suspected duplicates deleted by vigiMatch could be "false positives", i.e. reports that are not true duplicates, but have been marked as such or "false negatives", i.e. true duplicates that have not been highlighted by the algorithm leading to the disparities.

Duplicates could arise from same AEFI reports on vaccines submitted by different pharmaceutical companies and healthcare institutions (from multiple caregivers of patients and healthcare providers) to national 
pharmacovigilance centres. They could also arise via errors during the transfer of AEFI reports between different systems and databases. A thorough data audit therefore needs to be done before sending data to VigiAccess.

\section{Conclusion}

The study retrieved 27 categories of vaccine AEFIs from the VigiAccess website which involved all body systems. In all 813,973 AEFIs were obtained for all 8 studied vaccines from the search with general and vaccine administration site conditions being the highest number of AEFIs. The continental analysis yielded a total of 399,157 AEFIs out of which the Americas recorded the highest whereas Africa recorded the least. VigiAccess needs improvement in data synchronization to enhance its reliability.

\subsection{Recommendations}

1). Immunization programs in low and middle income countries must be strengthened and capacity for active surveillance and AEFI reporting improved. Capacity for active surveillance and AEFI could be built into existing health systems, particularly in countries with integrated electronic health information systems to make reporting easier.

2). All areas of immunization including pregnant mothers, infants, teenagers and adults must be improved to increase vaccination coverage.

3). All countries should be supported in the establishment of a process for causality assessment of serious AEFI to avoid misdiagnosis of AEFIs.

4). More rigorous efforts must be made by the Uppsala monitoring centre to reduce AEFI duplication as well as false negatives and positives in the VigiAccess database to repose more public confidence in VigiAccess data.

\section{Acknowledgements}

We acknowledge Dr. Didier Nzolo of the National Pharmacovigilance centre of the Democratic Republic of Congo for his valuable information on AEFI reporting pattern in 2015 from Vigibase. We are also grateful to the African Collaborating Centre for Advocacy and training in Pharmacovigilance, Accra Ghana for useful information on the Scope of Pharmacovigilance in Africa.

\section{Competing Interests Statement}

The authors declare that there is no conflict of interests regarding the publication of this paper.

\section{References}

Ampadu, H. H., Hoekman, J., de Bruin, M. L., Pal, S. N., Olsson, S., Sartori, D., ... Dodoo, A. N. O. (2016). Adverse Drug Reaction Reporting in Africa and a Comparison of Individual Case Safety Report

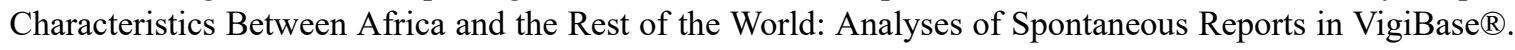
Drug Safety. https://doi.org/10.1007/s40264-015-0387-4

Aran, A., Lin, L., Nevsimalova, S., Plazzi, G., Hong, S. C., Weiner, ... Mignot, E. (2009). Elevated anti-streptococcal antibodies in patients with recent narcolepsy onset, Sleep. 32(8), 979-983.

Cashman, P., Macartney, K., Khandaker, G., King, C., Gold, M., \& Durrheima, D. N. (2017). Participant-centred active surveillance of adverse events following immunisation: A narrative review. International Health, 9(3), 164-176. https://doi.org/10.1093/inthealth/ihx019

Centres for Disease Prevention and Control. (2018). Vaccines and Preventable Diseases, Retrieved from https://www.cdc.gov/vaccines/vpd/vaccines-list.html

Chen, R. T., Shimabukuro, T. T., Martin, D. B., Zuber, P. L., Weibel, D. M., \& Sturkenboom, M. (2015). Enhancing vaccine safety capacity globally: a lifecycle perspective. Vaccine, 33(Suppl. 4), D46-54.

Chung, E. H., (2014). Vaccine allergies. Clinical and Experimental Vaccine Research, 3(1), 50-57. http://doi.org/10.7774/cevr.2014.3.1.50

Clothier, H. J., Selvaraj, G., Easton, H. L., Lewis, G., Crawford, N. W., \& Buttery, J. P. (2014). Consumer reporting of adverse events following immunization, Human Vaccines \& Immunotherapeutics, 10(12), 3726-3730.

Council for International Organizations of Medical Sciences, CIOMS. (2012). Definition and Application of Terms for Vaccine Pharmacovigilance, Report of CIOMS/WHO Working Group on Vaccine Pharmacovigilance, Geneva.

Crum-Cianflone, N. F., \& Wallace, M. R. (2014). Vaccination in HIV-Infected Adults. AIDS Patient Care and 
STDs, 28(8), 397-410. http://doi.org/10.1089/apc.2014.0121

Danova, J., Kocourkova, A., \& Celko, A. M. (2017). Active surveillance study of adverse events following immunisation of children in the Czech Republic. BMC Public Health, 17(1), 1-5. https://doi.org/10.1186/s12889-017-4083-4

Edwards, K. M. (2011). Causality Assessment of Serious Neurologic Adverse Events Following 2009 H1N1 Vaccination. Vaccine, 29(46), 8302-8308. http://doi.org/10.1016/j.vaccine.2011.08.093

Erlewyn-lajeunesse, M., Bonhoeffer, J., Ruggeberg, J. U., \& Heath, P. T. (n.d.). Following Immunisation, 737-739. https://doi.org/10.1136/jcp.2006.037457

Han, F., Lin, L., Warby, S. C., Faraco, J., Li, J., Dong, S. X., ... Mignot. (2011). Narcolepsy onset is seasonal and increased following the $2009 \mathrm{H} 1 \mathrm{~N} 1$ pandemic in China. Annals of neurology, 70(3), 410-417.

Han, J., Kemiki, O., Hsu, L. L., \& Rivers, A. E. (2015). Adverse Reactions to Pneumococcal Vaccine in the Pediatric and Adolescent Sickle Cell Patients. Pharmacotherapy, 35(7), 696-700. https://doi.org/10.1002/phar.1607

Hardt, K., Schmidt-Ott, R., Glismann, S., Adegbola, R. A., François, P., \& Meurice, F., P. (2013). Sustaining Vaccine Confidence in the 21st Century, Vaccines, 1, 204-224. https://doi.org/10.3390/vaccines1030204

Hazell, L., Cornelius, V., Hannaford, P., Shakir, S., \& Avery, A. J. (2013). Yellow Card Study Collaboration: How do patients contribute to signal detection?: a retrospective analysis of spontaneous reporting of adverse drug reactions in the UK's Yellow Card Scheme. Drug Safety, 36, 199-206. http://dx.doi.org/10.1007/s40264-013-0021-2

Hazell, L., \& Shakir, S. A. (2006). Under-reporting of adverse drug reactions: a systematic review. Drug Safety, 29, 385-396. http://dx.doi.org/10.2165/00002018-200629050-00003

Hu, Y., Li, Q., Lin, L., Chen, E., Chen, Y., \& Qi, X. (2013). Surveillance for adverse events following immunization from 2008 to 2011 in Zhejiang province, China. Clinical and Vaccine Immunology, 20(2), 211-217. https://doi.org/10.1128/CVI.00541-12

Inch, J., Watson, M. C., \& Anakwe-Umeh, S. (2012). Patient vs. healthcare professional spontaneous adverse drug reaction reporting: a systematic review. Drug Safety, 35, 807-818. http://dx.doi.org/10.1007/BF03261977

Isah, A. O., Pal, S. N., Olsson, S., Dodoo, A., \& Bencheikh, R. S. (2012). Specific features of medicines safety and pharmacovigilance in Africa. Therapeutic Advances in Drug Safety, 3(1), 25-34. https://doi.org/10.1177/2042098611425695

Lakemedelsverket Medical Products Agency. (2011). Occurrence of narcolepsy with cataplexy among children and adolescents in relation to the H1N1 pandemic and Pandemrix vaccinations. Results of a case inventory study by the MPA in Sweden during 2009 - 2010

Lamb, F., Ploner A., Fink, K., Maeurer, M., Bergman, P., Piehl, F., ... Dahlstrom, L. A. (2016). No Evidence for Disease History as a Risk Factor for Narcolepsy after A (H1N1) pdm09 Vaccination. PLoS ONE, 11(4), e0154296. https://doi.org/10.1371/journal.pone.0154296

Lei, J., Balakrishnan, M. R., Gidudu, J. F., \& Zuber, P. L. F. (2018). Use of a new global indicator for vaccine safety surveillance and trends in adverse events following immunization reporting 2000-2015. Vaccine, 36(12), 1577-1582. https://doi.org/10.1016/j.vaccine.2018.02.012

Li, R., McNeil, M. M., Pickering, S., Pemberton, M. R., Duran, L. L., Collins, L. C., ... Engler, R. J. M. (2014). Military Healthcare Providers Reporting of Adverse Events Following Immunizations to the Vaccine Adverse $\begin{array}{lllll}\text { Event Reporting } & \text { System. Military }\end{array}$ https://doi.org/10.7205/MILMED-D-13-00391

Linquist, M. (2008). VigiBase, the WHO Global ICSR Database System: Basic Facts. Therapeutic Innovation \& Regulatory Science, 42 (5), 409-419. https://doi.org/10.1177/009286150804200501

Lussier, N., Bourgault, A.-M., Gaudreau, C., \& Turgeon, P. (1999). A complication of BCG vaccine: A case of localized cutaneous abscess due to Mycobacterium bovis. The Canadian Journal of Infectious Diseases, 10(3), 257-259.

Miller, E. R., Moro, P. L., Cano, M., \& Shimabukuro, T. (2015). Deaths following vaccination: What does the evidence show? Vaccine, 33(29), 3288-3292. https://doi.org/10.1016/j.vaccine.2015.05.023

Miravalle, A., Biller, J., Schnitzler, E., \& Bonwit, A. (2010). Neurological complications following vaccinations, 
Neurological Research, 32(3), 285-92. https://doi.org/10.1179/016164110X12645013515214.

National Academy of Sciences (2013). The Childhood Immunization Schedule and Safety: Stakeholder Concerns, Scientific Evidence, and Future Studies. Retrieved 25th July, 2018, from https://www.ncbi.nlm.nih.gov/books/NBK206949/

Nzolo, D., Aloni, M. N., Ngamasata, T. M., Luemba, B. M., Marfeza, S. B., Ekila, M. B., ... Tona, N. L. (2013). Adverse events following immunization with oral poliovirus in Kinshasa, Democratic Republic of Congo: preliminary results. Pathogens and Global Health, 107(7), 381-384. https://doi.org/10.1179/2047773213Y.0000000113

Offit, P., A. (2005). The Cutter Incident: How America's first polio vaccine led to the growing vaccine crisis. Yale University Press: New Haven and London

Ozawa, S., \& Stack, M. L. (2013). Public trust and vaccine acceptance-international perspectives. Human Vaccines and Immunotherapeutics, 9(8), 1774-1778. https://doi.org/10.4161/hv.24961

Parrella, A., Braunack-Mayer, A., Gold, M., Marshall, H., \& Baghurst, P. (2013). Healthcare providers' knowledge, experience and challenges of reporting adverse events following immunisation: A qualitative study. BMC Health Services Research, 13, 313. http://dx.doi.org/10.1186/1472-6963-13-313

Parrella, A., Gold, M., Braunack-Mayer, A., Baghurst, P., \& Marshall, H. (2014). Consumer reporting of adverse events following immunization (AEFI): Identifying predictors of reporting an AEFI. Human Vaccines and Immunotherapeutics, 10(3), 747-754. https://doi.org/10.4161/hv.27459

Shankar, P. R. (2016). VigiAccess: Promoting public access to VigiBase. Indian Journal of Pharmacology, 48(5), 606-607. https://doi.org/10.4103/0253-7613.190766

Shimabukuro, T. T., Nguyen, M., Martin, D., \& DeStefano, F. (2015). Safety monitoring in the Vaccine Adverse Event Reporting System (VAERS), Vaccine, 33(36), 4398-4405. https://doi.org/10.1016/j.vaccine.2015.07.035

Uppsala Monitoring Centre (UMC) (2015). AEFI reporting in Vigibase. Retrieved from https://vigilyze.who-umc.org/ Dataset date (update): 02/06/2015

Uppsala Monitoring Centre [UMC] (2018). Members of the WHO program. Retrieved from https://www.who-umc.org/global-pharmacovigilance/members/

VigiAccess Q \& A. (n. d.). Report duplicates. Retrieved from http://www.vigiaccess.org/

Williams, S. E., Pahud, B. A., Vellozzi, C., Donofrio, P. D., Dekker, C. L., Halsey, N., ... Edwards, K.M. (2011). Causality assessment of serious neurologic adverse events following 2009 H1N1 vaccination, Vaccine, 29(46), 8302-8308.

Williams, S. E., Edwards, K. M., Baxter, R. P., LaRussa, P. S., Halsey, N. A., Dekker, C. L., ... Klein, N. P. (2013). Comprehensive assessment of serious adverse events following immunization by health care providers, Journal of Pediatrics, 162, 1276-1281.

World Health Organization. (2009). State of the world's vaccines and immunization (pp. 1-212). Retrieved from https://doi.org/10.4161/hv.6.2.11326

World Health Organization [WHO]. (2012a). Global vaccine safety blueprint The landscape analysis Global vaccine safety blueprint The landscape analysis Immunization, Vaccines and Biologicals. Retrieved from http://whqlibdoc.who.int/hq/2012/WHO_IVB_12.04_eng.pdf

World Health Organization [WHO]. (2012b). Global vaccine safety blueprint. Retrieved from http://extranet.who.int/iris/restricted/bitstream/10665/70919/1/

World Health Organization [WHO]. (2012c). Global vaccine safety blueprint, The landscape, Analysis, Retrieved from http://apps.who.int/iris/bitstream/10665/ 70854/1/WHO_IVB_12.04_eng.pdf

World Health Organization [WHO]. (2015). Performance indicators for vaccine safety monitoring systems. Retrieved from http://www.who.int/vaccine_safety/committee/topics/global_AEFI_monitoring/Dec_2014/en/

World Health Organization [WHO]. (2016). Global vaccine action plan, Secretariat annual report 2016. Retrieved from http://www.who.int/immunization/global_vaccine_action_plan/gvap_secretariat_report_2016.pdf?ua=1

World Population Review (2018). Continent and Region Population. Retrieved from http://worldpopulationreview.com/continents/ 
Yadav, S. (2008). Status of adverse drug reaction monitoring and pharmacovigilance in selected countries. Indian Journal of Pharmacology, 40(Supp11), S4-S9.

\section{Copyrights}

Copyright for this article is retained by the author(s), with first publication rights granted to the journal.

This is an open-access article distributed under the terms and conditions of the Creative Commons Attribution license (http://creativecommons.org/licenses/by/4.0/). 